\title{
Wireless Sensor Network Based Mobile Tracking System
}

\author{
Nyein Aye Maung Maung*, Makoto Kawai \\ Graduate School of Information Science and Engineering, Ritsumeikan University, 525-8577, Kusatsu, Shiga, \\ Japan. \\ * Corresponding author. Tel.:+818046444193; email: gr042065@ed.ritsumei.ac.jp \\ Manuscript submitted November 15, 2014; accepted May 12, 2015. \\ doi: 10.17706/ijcce.2016.5.1.31-40
}

\begin{abstract}
Mobile target tracking through large-scale wireless sensor networks (WSN) demands a large number of reference nodes, which is not feasible for resource-constrained WSNs. This paper proposes a resource-efficient mobile tracking system which integrates connectivity-based range-free approach and Received Signal Strength (RSS) based ranging approach to improve the tracking performance while reducing the specialized hardware requirement. In our proposed system, regulated hop-count values between static unknown nodes and reference nodes in the targeted area of interest for mobile tracking are first estimated in the network configuration phase. Then, location of the mobile target is tracked based on the regulated hop-count values and the available RSS measurements from its surrounding nodes at each tracking point without any extra hardware. Additionally, estimated location is enhanced by correcting with the known information of maximum velocity of the mobile target. Simulation and experimental results show that the proposed system offers preferable mobile tracking performance with minimum reference node utilization in both small-scale and large-scale networks.
\end{abstract}

Key words: Mobile tracking, WSN, regulated hop-count, RSS.

\section{Introduction}

Accurate and low-cost location tracking in WSNs plays an important role in applications like habitat monitoring, military surveillance and disaster relief. Existing WSN localization schemes can be categorized into two groups: range-free [1] and ranging or range-based [2] approaches. Range-free schemes determine the node's location by using connectivity information or number of hops (hop-count) between nodes without any specific hardware support. Although they offer lower accuracy than ranging approaches, they give low cost and less power consumption solutions. More precise location estimation can be achieved with ranging approaches which are typically based on RSS, time-of-arrival (TOA), time-difference-of-arrival (TDOA), or angle-of-arrival (AOA), but they require specialized hardware for ranging, thus consuming more power and cost which is not suitable for resource-constrained WSNs.

Recently, RSS has become a standard feature in most of the wireless devices without any extra hardware requirement. In fact, RSS-based mobile tracking approach becomes one of the most popular and cheap techniques. Typically, an RSS-based mobile tracking approach usually consists of a set of static nodes with known location information, referred to as reference nodes. The tracking algorithm tries to continuously estimate the mobile target's location from the RSS measurements between the mobile target and its neighboring reference nodes [3], [4]. Since existence of multipath fading, reflections, diffraction and interference highly affects the correspondence between the RSS and relative distance, more works [5], [6] 
are proposed to minimize the dynamic fluctuation of the radio signal received from each reference node.

However, existing RSS-based mobile tracking approaches require extensive channel calibrations and their applicability is limited to the targeted network environments. Significantly, at each tracking point, there must be at least three reference nodes inside the radio range of the mobile target. For tracking through a large-scale WSN, a high number of sensor nodes have to be attached with specialized localization hardware since manual configurations of locations for these nodes are not feasible for large-scale networks.

In this paper, we propose an efficient mobile tracking system which minimizes the utilization of reference nodes and improves the localization accuracy without any extra hardware. The main motivations and contributions of this paper are as follows.

1) Unlike existing works which need a large number of reference nodes for mobile tracking through large-scale WSNs, we propose an efficient solution which requires only three or four reference nodes.

2) Previous cost-effective tracking approaches utilize either connectivity information or RSS measurements to track the location of the mobile target. In contrast to this, we integrate connectivity information and RSS measurements in order to improve the location tracking accuracy.

3) An efficient location correction method is applied in the proposed system which shows significant improvement in the target tracking performance.

The rest of the paper is organized as follows. Section 2 presents the detailed workflow of the proposed mobile tracking system. Simulation and experimental based performance evaluations are discussed in Section 3 and finally in Section 4, conclusions and future considerations are described.

\section{Proposed Wireless Sensor Network Based Mobile Tracking System}

The proposed mobile tracking system is composed of two main phases, (i) network configuration phase and (ii) real-time mobile tracking phase. There are three different node types in the proposed system: mobile target, reference or anchor node with fixed prior location information, and assistant node whose location is unknown but supports packet communications and data processing.

\subsection{Network Configuration Phase}

In this phase, an effective network configuration strategy is proposed to reduce the number of reference nodes required and to get the useful information for further mobile tracking phase. We set four reference nodes at the corners of the deployment area and low-cost sensor nodes with no location-aided hardware (assistant nodes) are scattered into the area of interest.

Then, connectivity information of the network is configured using the optimal RSS threshold based connectivity configuration proposed in our previous work [7] to make the proposed system robust to any network size (small to large-scale network scenarios). Each node $i$ in the network broadcasts $t$ packets from which each receiving node $k_{j}\left(j=1,2, \ldots, N_{t}\right.$ where $N_{t}$ is the total number of nodes in $i$ 's communication range) measures the RSS values. A set of RSS samples between nodes $i$ and $k_{j}$ are collected and the mean RSS value of these samples $\left(R S S_{i j}\right)$ is computed. Then, only those pair of nodes whose $R S S_{i j}$ value exceeds the predefined optimal RSS threshold $\left(R S S_{\text {th-opt }}\right)$ is considered as connected and regarded as neighbors.

$$
C_{i j}=\left\{\begin{array}{l}
1, R S S_{i j} \geq R S S_{t h-o p t} \text { (connected) } \\
0, R S S_{i j}<R S S_{t h-o p t} \text { (not-connected) }
\end{array}\right.
$$

$R S S_{\text {th-opt }}$ for different number of nodes and the network size is derived using (2) [7].

$$
R S S_{\text {th-opt }}=P_{\text {ref }}-10 n \log _{10} R_{0}
$$


where $P_{\text {ref }}$ is the reference power measured at $1 \mathrm{~m}, n$ is the pathloss exponent and $R_{0}$ is the minimum radio range required to avoid isolated nodes and to achieve a connected wireless network derived using (3).

$$
R_{0}=\sqrt{-\ln \left(1-p^{1 / N_{\text {total }}}\right) / \phi \pi}
$$

where $p$ is the probability that no node in the network is isolated and $\varphi$ is the node density $\left(\varphi=N_{\text {total }} /\right.$ Area $)$. $N_{\text {total }}$ is the total number of nodes in the network and Area is the deployment area, both of which are the prior information in most cases of practical interest of localization.

After configuration the connectivity information, regulated hop-count $(R H)$ values among reference nodes and assistant nodes are estimated for further mobile tracking process. First, each reference node $r_{f}$ $(f=1,2, \ldots, G$ where $G$ is the total number of reference nodes) broadcasts a packet containing its own ID, its location and the hop-count value initialized to zero. Each intermediate node $u$ determines whether the sender node $j$ is its neighbor or not using the optimal RSS threshold based connectivity information. If node $j$ is defined to be neighbor of node $u$ (i.e. $C_{u j}=1$ ), $u$ calculates the $R H$ value between $u$ and $j\left(R H_{u j}\right)$ using (4).

$$
R H_{u j}=d_{u j} / R_{0}
$$

where $d_{u j}$ is the RSS-based distance between nodes $u$ and $j$ which is calculate based on the log-normal shadowing model equation [8],

$$
d_{u j}=10 \frac{P_{r e f}-R S S_{u j}+X_{\sigma}}{10 n}
$$

where $R S S_{u j}$ is the mean RSS value between nodes $u$ and $j$ obtained from the connectivity configuration stage and the shadowing factor $X_{\sigma}$ of 0 is set. After that, $u$ finds the $R H$ value to the reference node ID in the received packet by adding $R H_{u j}$ with the hop-count value in the packet. If the new hop-count value is larger than old value stored at $u$ for that reference node, $u$ simply ignores the packet. Else, old value is replaced with the new value and $u$ forwards the packet with the new hop-count value. Through this mechanism, all assistant nodes have the minimum $R H$ values to all the reference nodes which will be utilized in the real-time mobile tracking phase. As well, each reference node estimates the average size of one hop (hop-distance value) by dividing total distances to other reference nodes with total $R H$ values to them, and informs it to the centralized computer. With traditional range-free schemes, if the two nodes are regarded as neighbors, distance information between them is regarded as 1-hop (i.e. size of the radio range) although their Euclidean distances are different. In contrast to this, more precise proximity information can be obtained by using the proposed regulated hop-count values. This network configuration phase has to be carried out only once while configuring the targeted area of interest for mobile tracking process.

\subsection{Real-Time Mobile Tracking Phase}

The proposed mobile tracking algorithm tries to locate successive movements of the mobile target by making use of the information from the surrounding static wireless sensor nodes. Before going into details of the algorithm, let us formulate some of the packet types used: (i) $R Q$ : packet transmitted by the mobile target to request information from its neighbors, (ii) $R P$ : packet transmitted by the neighbors of the mobile target to respond the $R Q$ packet and (iii) Loc: packet transmitted to inform the estimated location of the mobile target to the centralized server. 


\subsubsection{Information aggregation}

This section describes how necessary information for tracking the mobile target is aggregated. The mobile target broadcasts an $R Q$ packet on each specific period $\Delta T\left(\Delta T=T_{i}-T_{i-1}\right.$ where $T_{i}$ is the current tracking time and $T_{i-1}$ is the previous tracking time). $R Q$ contains packet type, mobile target's ID and $T_{i \text {. }}$

Each node $n_{b}$ receiving the $R Q$ packet at each tracking point $\left(b=1,2, \ldots, N_{i}\right.$ where $N_{i}$ is the total number of nodes in the radio range of mobile target at time $T_{i}$ ) measure the RSS value of the incoming packet. If the measured RSS value is above the optimal RSS threshold value, $n_{b}$ regards the mobile target $m$ as its neighbor and transmits $R P$ packet to the mobile target $m$. If below the threshold, $n_{b}$ ignores the request. $R P$ packet contains the packet type, $T_{i}$ in the corresponding $R Q$ packet, mobile target ID, measured RSS value between the mobile target $m$ and $n_{b}\left(R S S_{m b}\right)$, and the $R H$ values between $n_{b}$ and all reference nodes $\left(R H_{b f}\right.$ where $f=1,2, \ldots, G$ ) obtained from the network configuration phase.

\subsubsection{Location estimation}

When $m$ receives $R P$ packets, it calculates the $R H$ values between it and each neighbor $n_{b}\left(b=1,2, \ldots, N_{i_{-} \text {thld }}\right.$ where $N_{i_{-} \text {thld }}$ is the number of $R S S_{\text {th-opt }}$ based neighbors) based on the RSS value in the RP packet.

$$
R H_{m b}=d_{m b} / R_{0},
$$

where $d_{m b}$ is the RSS-based distance between $m$ and $n_{b}$ using (5). After that, the regulated hop-count value between the mobile target $m$ and each reference node $r_{f}\left(R H_{m f}\right.$ where $\left.f=1,2, \ldots, G\right)$ is calculated using (7).

$$
\begin{gathered}
R H_{m f}=\min (m f(b)) \\
m f(b)=R H_{m b}+R H_{b f} \text { where } b=1,2, \ldots, N_{i_{-} \text {thld }}
\end{gathered}
$$

Then, distance from the mobile target $m$ to each reference node is estimated by multiplying $R H_{m f}$ with the hop-distance value of the nearest reference node. Finally, lateration method is applied to get location information of the mobile target at $T_{i}$.

After calculating the location of the mobile target using the regulated hop-count values, the next step is to correct the estimated location using the known information of maximum velocity of the mobile target $\left(V_{\max }\right)$. From $V_{\max }$, possible maximum movement $\left(S_{\max }\right)$ during each sampling period $\Delta T$ can be estimated as

$$
S_{\text {max }}=V_{\text {max }} \times \Delta T
$$

Suppose that estimated location of the mobile target at time $T_{i}$ is $\left(x_{i}, y_{i}\right)$ and $T_{i-1}$ is $\left(x_{i-1}, y_{i-1}\right)$, the movement between $T_{i}$ and $T_{i-1}$ can be obtained by using (10),

$$
S=\sqrt{\left(x_{i}-x_{i-1}\right)^{2}+\left(y_{i}-y_{i-1}\right)^{2}}
$$

Then, we derive the correction vector $V_{t}$ using (11).

$$
V_{t}= \begin{cases}\left(x_{i}-x_{i-1}, y_{i}-y_{i-1}\right) \times\left[\frac{S_{\max }-S}{S}\right] & , \text { if }\left(S>S_{\max }\right) \\ (0,0) & , \text { if }\left(S \leq S_{\max }\right)\end{cases}
$$


After that, location of the mobile target at time $T_{i}$ is updated using

$$
\left(x_{i}, y_{i}\right)=\left(x_{i}, y_{i}\right)-V_{t} \text {. }
$$

After getting the updated location at time $T_{i}$ using (12), the mobile target transmits $L o c$ packet to the centralized computer which contains its estimated location. Simplified algorithm of the proposed mobile tracking algorithm is illustrated in Algorithm 1. With the proposed algorithm, the total number of packet communications required to estimate the location of mobile target at time $T_{i}$ is

$$
N_{p k t}(i)=1+N_{i \_t h l d}+\text { hops }
$$

where hops is the number of hops Loc packet has to transverse to reach the centralized computer.

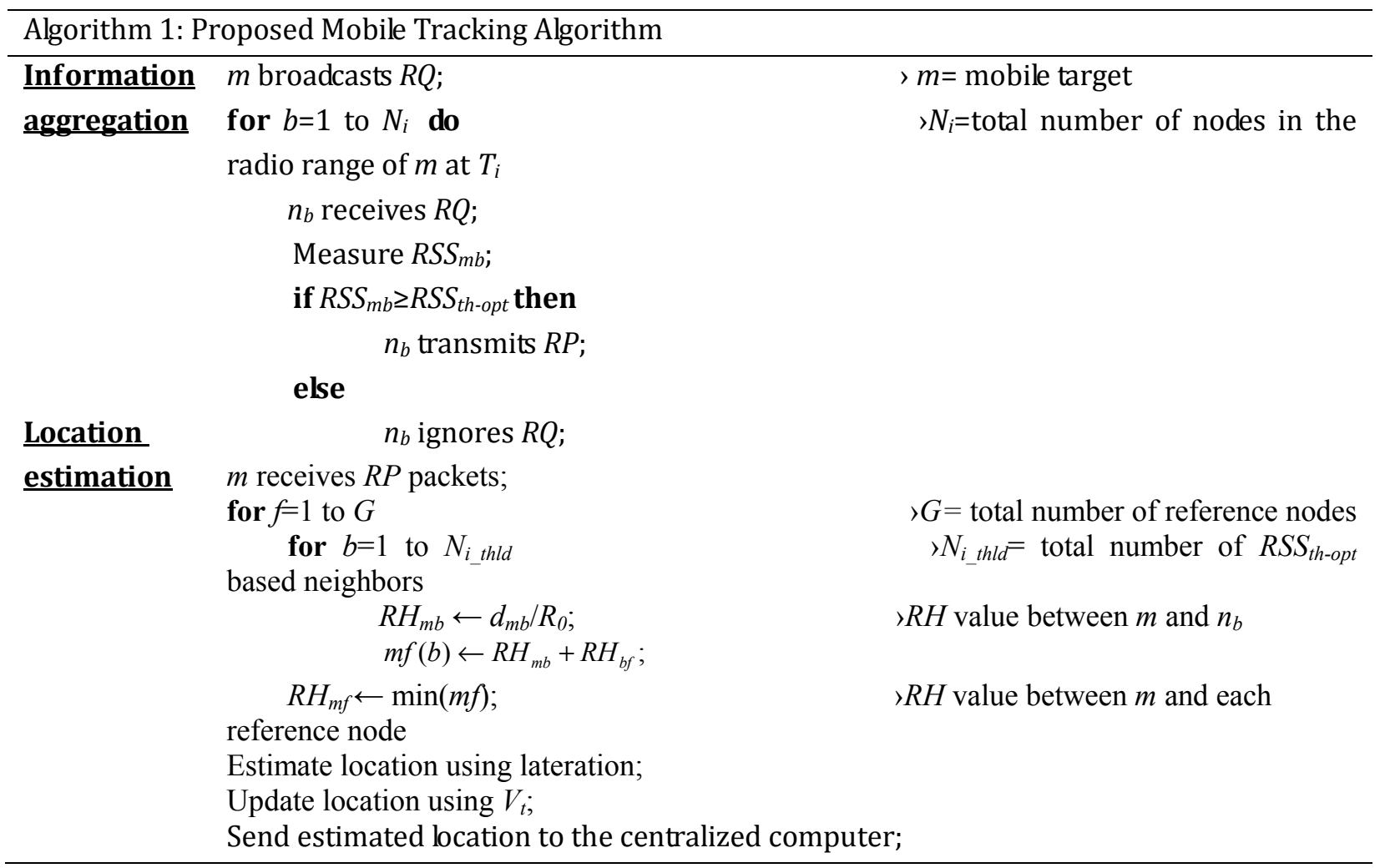

\section{Performance Evaluations}

Performance of the proposed mobile tracking system is evaluated through simulations in MATLAB environment and real world experiments. The following mean error value is used as a localization accuracy evaluation function,

$$
\text { mean error }=\frac{1}{N} \sum_{i=1}^{N}\left(\sqrt{\left(x-x_{i}\right)^{2}+\left(y-y_{i}\right)^{2}} / R\right)
$$

where $N$ is the total number of tracking points, $R$ is the radio range, $\left(x_{i}, y_{i}\right)$ and $(x, y)$ are the estimated location and the actual location of the mobile target at tracking point $i$ respectively. To implement the proposed system in practice, we use XBee Series 2 modules [9] which are based on IEEE 802.15.4 ZigBee Standard to build the WSN. These modules have the ability to measure the RSS value of incoming packet without any extra hardware. Wireless modules are mounted on Arduino UNO and Arduino wireless shields. 
For the mobile target, we use Bioloid obstacle detection robot [10] which has the maximum velocity $\left(V_{\max }\right)$ of $0.067 \mathrm{~ms}^{-1}$. Data rate of $38400 \mathrm{kbps}$, packet sizes of 20, 37 and 28 bytes for $R Q, R P$ and Loc packets respectively are used in our experiments. Effective radio range $(R)$ of XBee Series 2 modules for NLOS environment is $40 \mathrm{~m}$. The same parameters are utilized in the simulations to reflect the real world environment. Firstly, two sets of simulations are carried out on a $200 \times 200 \mathrm{~m}^{2}$ large-scale area, (i) Test 1 : 121 nodes grid topology network and (ii) Test 2: 121 nodes random topology network. Centralized computer is placed at the bottom right corner of the deployment areas. RSS values are modeled using the log-normal shadowing model with the radio propagation characteristics of pathloss exponent of 2.6 and standard deviation of 6.2, assuming indoor NLOS environment [7]. The movement of the mobile target is simulated to follow the square shape moving path. Speed of the node is chosen randomly between 0 and $V_{\max }$. We use sampling frequency $\Delta T$ of $1 \mathrm{~Hz}$ in all our simulations and experiments.

Fig. 1 and Fig. 2 illustrate the performance comparison between the proposed system and the RSS-based mobile tracking approach for Tests 1 and 2. With the proposed system, only four reference nodes denoted with triangles $(\Delta)$ are placed at the corners and assistant nodes denotes with small circles $(\bullet)$ are distributed inside. With the RSS-based tracking approach, all nodes in the network are assigned with the prior location information (reference nodes). Blue line represents the actual mobility path of the target and green line represents the estimated result. Results in sub-figures (a) and (b) indicate that although tracking results of the proposed system without updating with the correction vector $V_{t}$ and updating with $V_{t}$ give similar accuracy, the later offers smoother tracking path. Moreover, the proposed scheme shows better localization accuracy than the RSS-based approach since we effectively integrate the connectivity-based localization and the RSS ranging to minimize the RSS variability problem and to improve the accuracy. Significantly, only four reference nodes are necessary with our proposed system whereas all nodes have to be reference nodes which require additional location-aided hardware with the RSS-based approach.

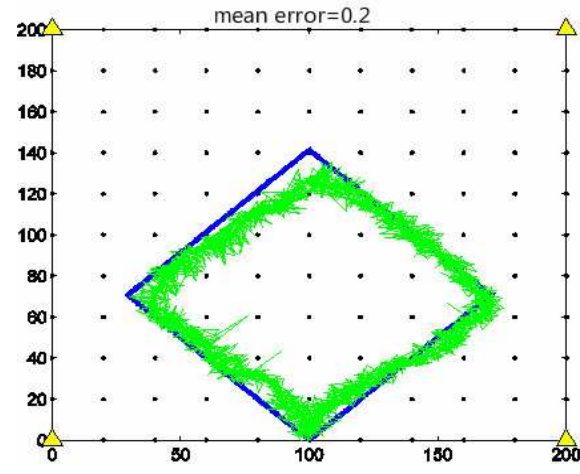

(a) Proposed system (without correction).

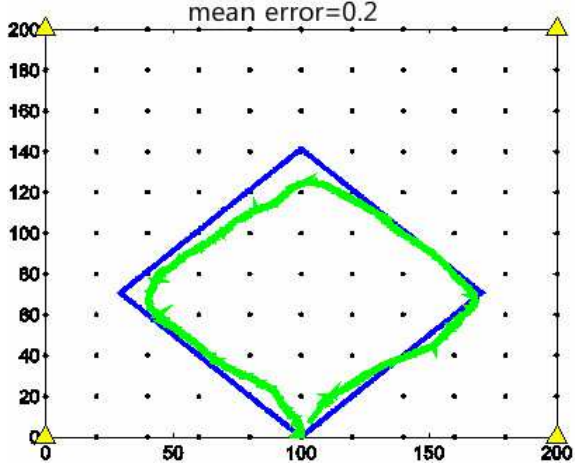

(b) Proposed system (with correction).

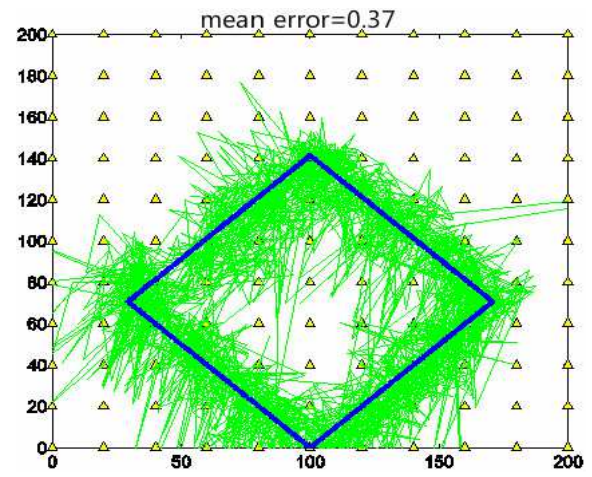

(c) RSS-based approach.

Fig. 1. Mobile tracking performance for grid topology network (Test 1). 

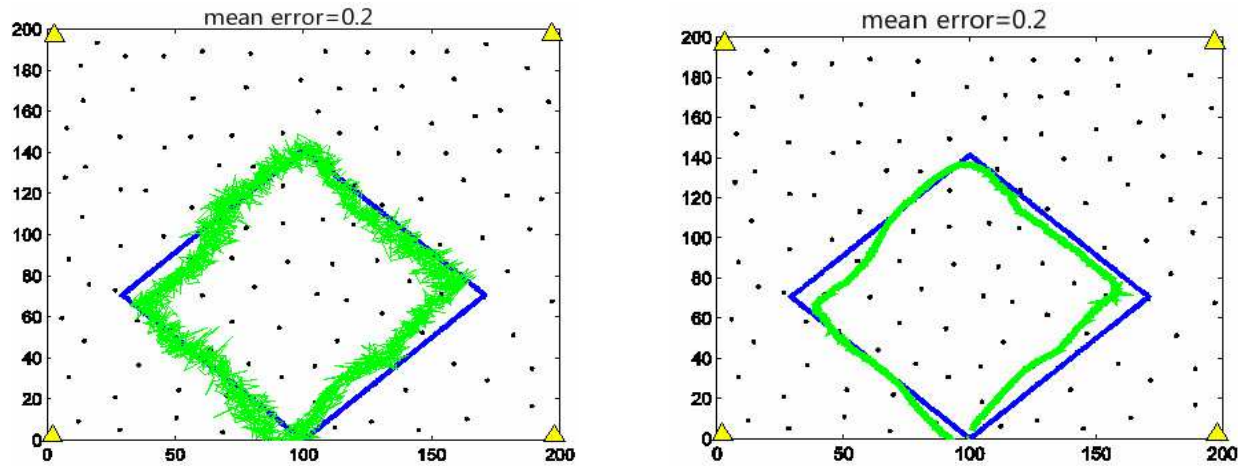

(a) Proposed system (without correction). (b) Proposed system (with correction).

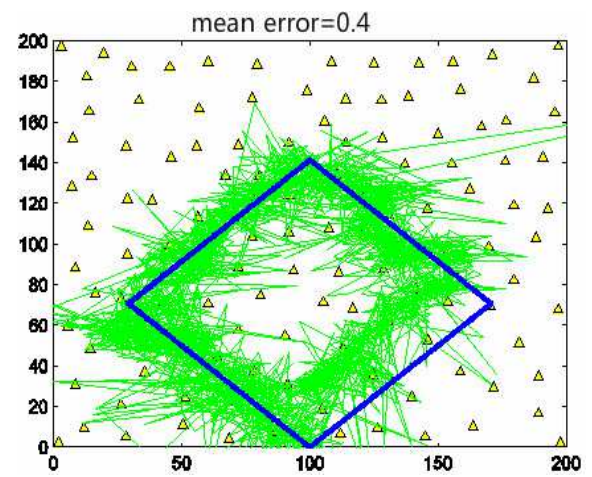

(c) RSS-based approach.

Fig. 2. Mobile tracking performance for random topology network (Test 2).

In addition, to evaluate the performance of the proposed scheme and the RSS-based tracking approach for low density network scenario, simulations are conducted on a grid topology network with 36 nodes. As shown in Fig. 3, the proposed scheme still shows preferable performance with very low density network since location of the mobile target at each tracking point can be estimated even with information from only one neighbor. But with the RSS-based approach, at some points of the actual moving path, denoted with red color in Fig. 3(b), there is no enough reference nodes for localization process (at least 3 reference nodes are required) and the mobile target cannot be tracked at those points.

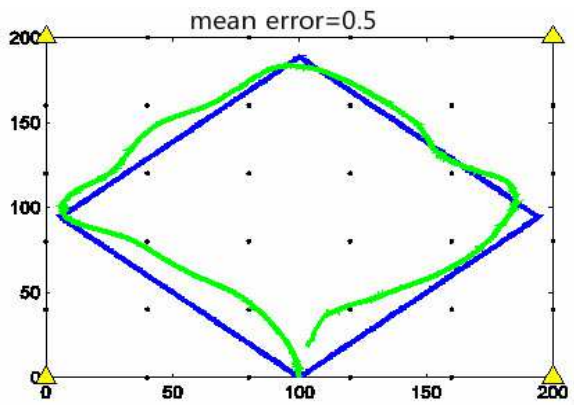

(a) Proposed system.

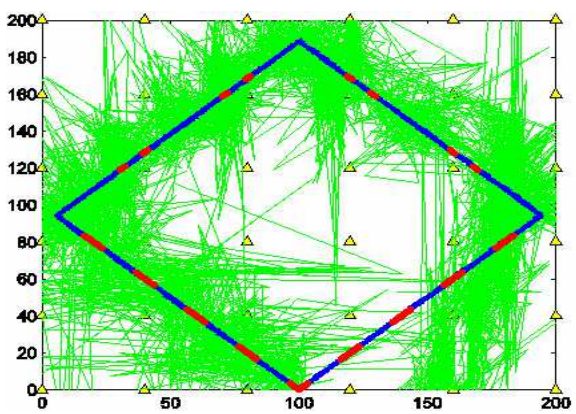

(b) RSS-based approach.

Fig. 3. Mobile tracking performance for low density network.

Next, communication costs of the proposed system and the RSS-based tracking method to track the location of the mobile target through a large-scale WSN are evaluated. Simulations are conducted on a $200 \times 200 \mathrm{~m}^{2}$ random network topology with 200 nodes. Required packet communications for tracking the mobile target at $T_{i}$ using the proposed scheme is calculated using (13), and the RSS-based tracking approach is calculated using (15). 


$$
\mathrm{N}_{\mathrm{pkt}}(i)=1+N_{i}+\text { hops }
$$

Then, average communication cost is calculated using (16),

$$
\text { AvgPkts }=\frac{1}{N} \sum_{i=1}^{N} N_{p k t}(i) \text {. }
$$

Fig. 4 presents the tracking accuracy and the average communication cost for different radio range levels. For all radio range levels, the same tracking period is applied. Larger radio range level gives higher number of surrounding neighbors $\left(N_{i}\right)$ which can receive the $R Q$ packet from the mobile target at each point. Hence, the RSS-based mobile tracking approach shows higher communication cost with increasing the radio range levels. In contrast to this, changing the radio range level has no impact on the performance of the proposed system since only the nodes which are defined to be connected to the mobile target using the optimal RSS threshold based connectivity configuration participate in the tracking process for any radio range level.

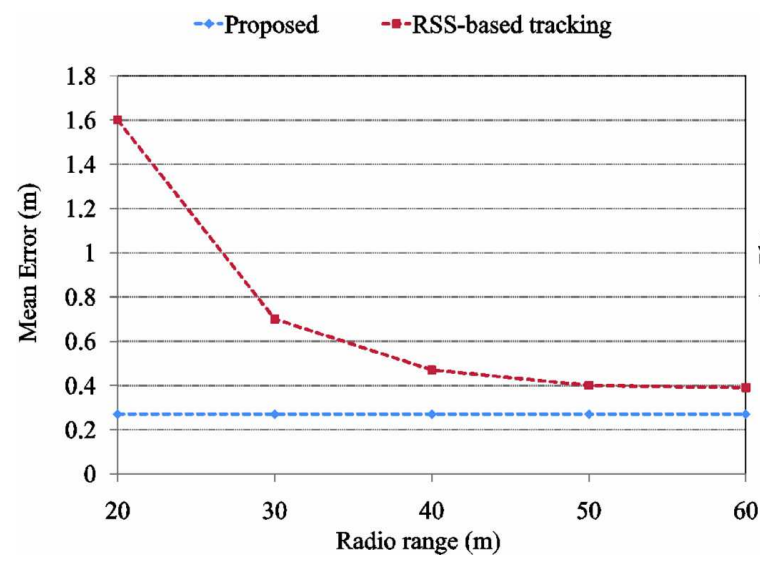

(a) Accuracy

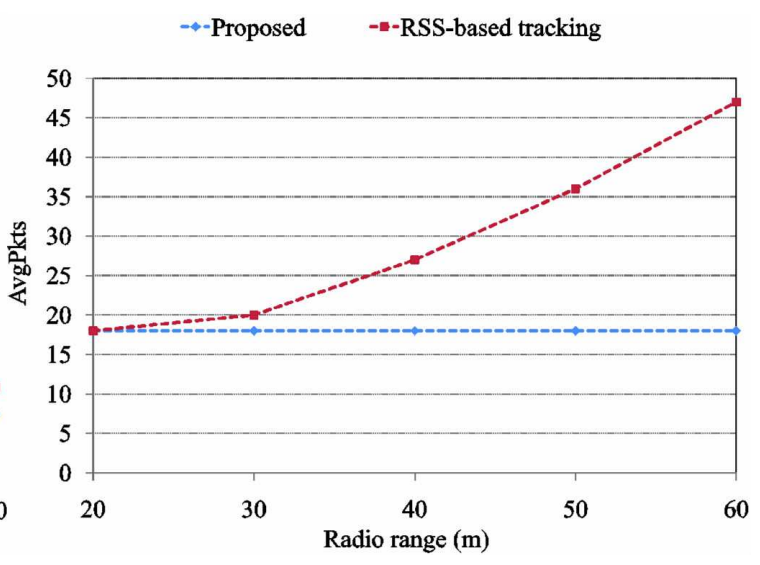

(b) Communication cost

Fig. 4. Performance for different radio range levels.

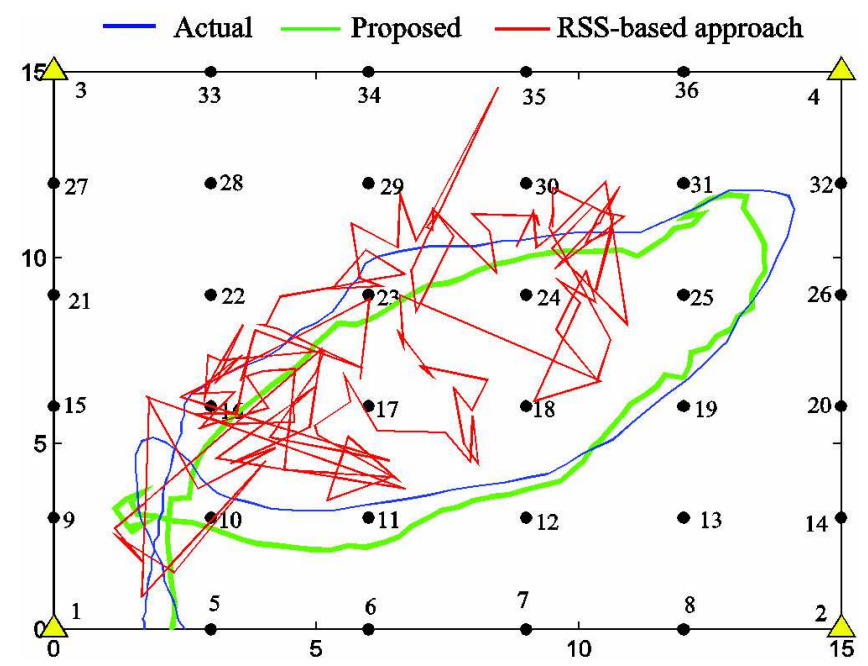

Fig. 5. Performance comparison for small-scale network.

To evaluate the performance of the proposed system on small-scale WSNs, experiments are conducted in Ritsumeikan University gymnasium. Four reference nodes are placed at the corners of the $15 \times 15 \mathrm{~m}^{2}$ deployment area, 32 assistant nodes are distributed over the tracking area of interest and one XBee module 
is attached on the Bioloid robot. The centralized computer is within the radio range of the mobile target and its surrounding nodes at each tracking point since XBee Series 2 modules cover $40 \mathrm{~m}$ radio range level even in NLOS environment. To minimize the computational overheads on the mobile target, we make some changes in the proposed mobile tracking algorithm that neighbors of the mobile target transmit $R P$ packets directly to the centralized computer, without transmitting them back to the mobile target. Then, centralized computer performs the location estimation process using the received information. Results in Fig. 5 indicate that tracking performance of the RSS-based approach is largely affected by the high RSS variability in practical environment, whereas our proposed system offers preferable tracking performance.

Finally, we evaluate the localization delay (the time it takes from $R Q$ packet transmission until the result is obtained at the centralized computer) of the proposed system. The localization delay $\left(D_{i}\right)$ at each tracking point $i$ for large-scale and small-scale networks are

$$
\begin{gathered}
D_{i}(\text { large-scale })=T_{r q}+A_{p r o c}+\left(N_{i_{-} t h l d} \times T_{r p}\right)+M_{p r o c}+\left(\text { hops } \times T_{l o c}\right) \\
D_{i}(\text { small-scale })=T_{r q}+A_{p r o c}+\left(N_{i_{-} t h l d} \times T_{r p}\right)
\end{gathered}
$$

where $T_{r q}, T_{r p}$ and $T_{l o c}$ are packet delivery time for $R Q, R P$ and $L o c$ packets respectively. $A_{p r o c}$ is the processing time on each neighbor of the mobile target and $M_{\text {proc }}$ is the processing time on mobile target to calculate the estimated location. We neglect the processing time on the centralized computer. Based on the experimental parameters, average $D_{i}$ for tracking the mobile target through the small-scale network shown in Fig. 5 is $0.09 \mathrm{~s}$. It means that estimated location of the mobile target at sampling time $T_{i}$ is displayed at the centralized computer after the mobile target has already moved by $0.006 \mathrm{~m}$. A larger localization delay will be resulted with multi-hop large-scale networks since Loc packet has to transverse several hops to reach the centralized computer. To minimize the localization delay, wireless modules with higher processing capability micro-controllers should be utilized for tracking through large-scale networks.

\section{Conclusions}

In this paper, we propose an efficient WSN-based mobile tracking system which not only improves the localization accuracy but also reduces the extra hardware requirement by integrating the connectivity-based range-free and the RSS-based ranging approaches. The mobile target is tracked based on the regulated hop-counts values and the available RSS measurements from the surrounding nodes, instead of using the known location information of them, to minimize the reference node utilization. Additionally, known information of the maximum velocity of the mobile target is effectively applied to improve the performance of the proposed tracking algorithm. Results show that the proposed system shows preferable tracking performance in both small and large-scale networks with different node densities. Multiple mobile targets can be tracked using the proposed system, but considerations of reducing communication overheads due to multiple targets will be future extension to our current work. Proposed system can be deployed in many applications like tracking rescuers or victims in case of disaster relief and tracking employees in workspace.

\section{References}

[1] He, T., et al. (2003). Range-free localization schemes for large scale sensor networks. Proceedings of the Ninth Annual International Conference on Mobile Computing and Networking. San Diego, California.

[2] Chaurasia, S. (2011). Analysis of range-based localization schemes in wireless sensor networks: A statistical approach. Proceedings of International Conference on Advanced Communication Technology. 
Seoul.

[3] Blumrosen, G., et al. (2013). Enhancing RSSI-based tracking accuracy in wireless sensor networks. ACM Transactions on Sensor Networks, 9(3).

[4] Alhmiedat, T. A., \& Yang, S. H. (2008). A ZigBee-based mobile tracking system through wireless sensor networks. International Journal of Advanced Mechatronic Systems, 1(1), 63-70.

[5] Lau, E. E. L., et al. (2008). Enhanced RSSI-based high accuracy real-time user location tracking system for indoor and outdoor environments. International Journal on Smart Sensing and Intelligent Systems, 1(2), 534-548.

[6] Bertinato, M., et al. (2008). RF Localization and tracking of mobile nodes in wireless sensors networks: Architectures, algorithms and experiments. Technical Report, University of Padova.

[7] Maung, N. A. M., \& Kawai, M. (2014). Performance improvement of connectivity-based localization using iterative learning. Proceedings of International Conference on Localization and GNSS. Helsinki, Finland.

[8] Rappaport, T. S. (2002). Wireless Communications: Principles and Practice (2nd ed.). Prentice Hall.

[9] XBee ${ }^{\circledR Z B}$. From http://www.digi.com/products/wireless-wired-embedded-solutions/zigbee-rf-modules/ zigbee-mesh-module/xbee-zb-module

[10] BIOLOID. Form http://www.robotis.com/xe/BIOLOID_main_en

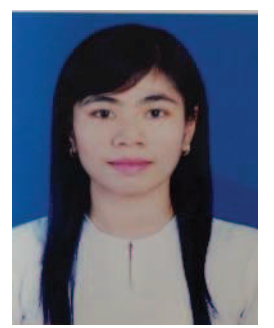

Nyein Aye Maung Maung received her B.E. and M.E. degrees in information technology from Yangon Technology University, Yangon, Myanmar, in 2004 and 2006 respectively. She joined Ritsumeikan University in 2006, and received the M.E degree in the field of advanced information science and engineering in 2008. From 2008 to 2011, she worked as an assistant lecturer in Yangon Technological University, Yangon, Myanmar. She is currently pursuing the doctoral degree course at Graduate School of Information Science and Engineering, Ritsumeikan University, Shiga, Japan. Her research interests include communication protocols and localization of wireless ad-hoc and sensor networks.

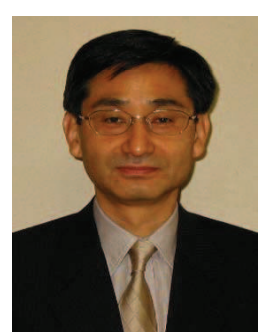

Makoto Kawai received the B.S., M.S. and Ph.D. degrees from Kyoto University, Kyoto, Japan in 1972, 1974 and 1987, respectively. During 1974 and 1999, he worked mainly for research and development of satellite and wireless communication systems at Nippon Telegraph and Telephone Corporation (NTT). He was an associate professor at Graduate School of Informatics, Kyoto University from 1999 to 2003. He was a professor of College of Science and Engineering from 2003 to 2004 and is a professor of College of Information Science and Engineering from 2004 to now in Ritsumeikan University, Shiga, Japan. His current research interests include satellite communication systems, and communication protocols and localization issues in wireless networks. He is a fellow of the IEICE and a member of AIAA, IEEE and IPSJ. 